\title{
Assessing the feasibility of applying a combined osteopathy and psychoeducation therapy (OsteoPeCT) and its influence on levels of psychological and physiological stress in adult participants with moderate stress: A pilot study
}

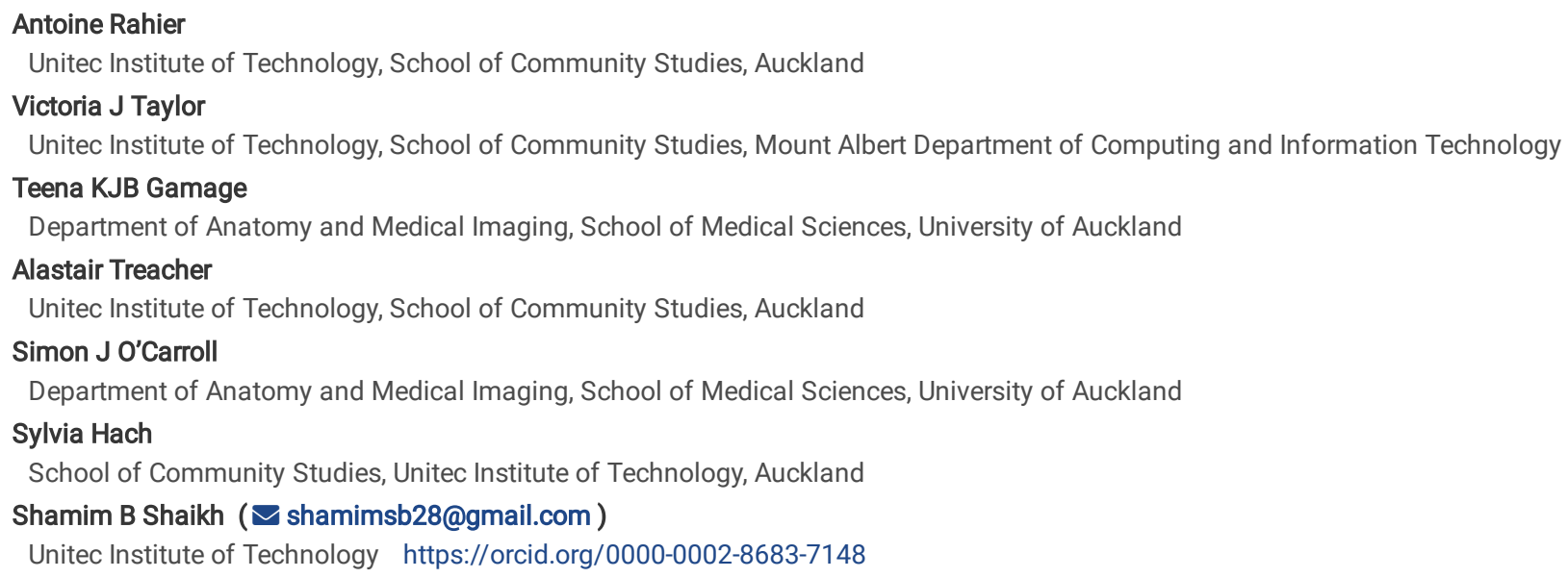




\section{Abstract}

Background: There is mounting evidence suggesting a relationship between stress and adverse health outcomes. Stress is a multidimensional phenomenon requiring a multimodal approach. While there is some evidence indicating a positive effect of massage therapy, there is limited research regarding the impact of related approaches such as general osteopathic techniques (GOTs). Further, research examining the feasibility and effectiveness of combining GOTs with psychoeducation in the management of stress is lacking. The present pilot study aimed to assess the feasibility of applying a therapy package consisting of GOTs and brief psychoeducation and its influence on moderate stress in a convenience sample.

Methods: A pilot uncontrolled trial with mixed pragmatic and exploratory design was conducted. The therapy package comprised of ten GOTs and ten minutes of scripted psychoeducation (OsteoPeCT) was applied in two sessions over two consecutive days to 18 adult participants with moderate stress. Feedback from participants and challenges experienced by both participants and researchers were recorded. The effects of OsteoPeCT were assessed by measuring preand post- intervention scores of self-reported perceived stress (Perceived Stress Scale-10, PSS-10; Profile of Mood Scale, POMS) and salivary levels of physiological stress biomarkers (cortisol; secretory immunoglobulin A, slgA and interleukin-6, IL-6).

Results: All aspects related to the application of OsteoPeCT (participant recruitment, participant retention, therapy application, administration of health screen and self-reported perceived stress questionnaires, and the collection of saliva samples for biomarker analysis) were feasible. A total of 18 participants were enrolled. The timing of sessions on consecutive days was reported to be challenging. While a measurable decrease in perceived stress (PSS-10) and in mood scores (Tense, Fatigue, Depression, Anger) were noted post therapy (OsteoPeCT) application, physiological stress markers were unaffected. Diurnal variations of these biomarkers may need further consideration.

Conclusion: The application of OsteoPeCT was feasible, well received with some beneficial influence on perceived stress indicating that an integration of psychoeducation and osteopathic care may confer benefits to patients. Future investigations with adapted protocols and larger sample size is warranted to assess effectiveness.

Trial registration: Retrospectively registered in Australian New Zealand Clinical Trials Registry (registration number ACTRN12620000763943 ) and ICTRP .

\section{Full Text}

This preprint is available for download as a PDF.

\section{Tables}

Table 1-Inclusion and exclusion criteria for participant recruitment

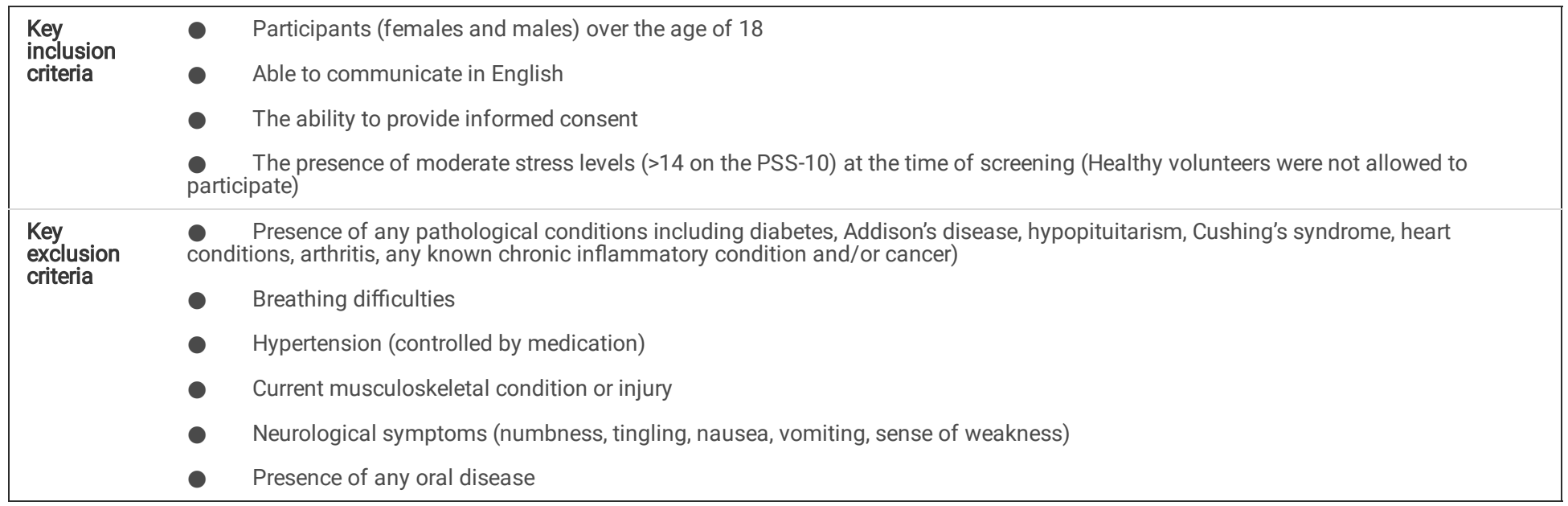

PSS-10 =Perceived Stress Scale-10

Table 2 - Participant characteristics 


\begin{tabular}{|c|c|c|c|c|c|c|c|c|c|c|c|}
\hline $\begin{array}{l}\text { Participant } \\
\text { ID number } \\
\text { (\#) }\end{array}$ & Sex & Age & $\begin{array}{l}\text { Marital } \\
\text { status }\end{array}$ & $\begin{array}{l}\text { Blood } \\
\text { pressure } \\
(\mathrm{mmHg})\end{array}$ & $\begin{array}{l}\text { Heart rate } \\
(\mathrm{bpm})\end{array}$ & $\begin{array}{l}\text { Height } \\
\text { (cm) }\end{array}$ & Weight (kg) & $\begin{array}{l}\text { Body } \\
\text { Mass } \\
\text { Index } \\
(\mathrm{BMI}) \\
\left(\mathrm{kg} / \mathrm{m}^{2}\right)\end{array}$ & $\begin{array}{l}\text { Systems Review } \\
\text { (presence/indication } \\
\text { of any issues) }\end{array}$ & $\begin{array}{l}\text { General } \\
\text { Health }\end{array}$ & $\begin{array}{l}\mathrm{Ne} \\
\mathrm{Sy}:\end{array}$ \\
\hline 1 & $\mathrm{~F}$ & 31 & $\mathrm{~s}$ & $122 / 82$ & 82 & 168 & 67 & 23.7 & $\mathrm{~N}$ & $\mathrm{~N}$ & $\mathrm{~N}$ \\
\hline 2 & $\mathrm{~F}$ & 22 & $\mathrm{R}$ & $111 / 69$ & 61 & 158 & 63 & 25.2 & $\mathrm{~N}$ & $\mathrm{~N}$ & $\mathrm{~N}$ \\
\hline & $\mathrm{F}$ & 23 & $\mathrm{R}$ & $112 / 68$ & 78 & 167 & 63 & 22.6 & Y & $\mathrm{N}$ & Y \\
\hline 3 & & & & & & & & & (Fungal infection) & & \\
\hline 4 & $\mathrm{~F}$ & 27 & Md & $110 / 74$ & 56 & 182 & 66 & 19.9 & $\mathrm{~N}$ & $\mathrm{~N}$ & $\mathrm{~N}$ \\
\hline 5 & $\mathrm{~F}$ & 51 & Md & $118 / 84$ & 79 & 157 & 51 & 20.7 & Y & $\mathrm{N}$ & $\mathrm{N}$ \\
\hline & & & & & & & & & (Fungal infection) & & \\
\hline 6 & $\mathrm{~F}$ & 58 & Md & $118 / 89$ & 78 & 177 & 78 & 24.9 & $\begin{array}{l}\text { Y (Musculoskeletal } \\
\text { injury) }\end{array}$ & $\mathrm{N}$ & $\mathrm{N}$ \\
\hline 7 & $\mathrm{~F}$ & 21 & S & $116 / 80$ & 62 & 170 & 68 & 23.5 & $\mathrm{~N}$ & $\mathrm{~N}$ & $\mathrm{~N}$ \\
\hline 8 & $\mathrm{~F}$ & 28 & Md & $110 / 75$ & 51 & 165 & 72 & 26.4 & $\mathrm{~N}$ & $\mathrm{~N}$ & $N$ \\
\hline 9 & $\mathrm{~F}$ & 28 & $\mathrm{Md}$ & $120 / 81$ & 41 & 176 & 70 & 22.6 & $\mathrm{~N}$ & $\mathrm{~N}$ & $\mathrm{~N}$ \\
\hline \multirow[t]{2}{*}{10} & $\mathrm{~F}$ & 53 & D & $117 / 77$ & 76 & 172 & 78 & 26.4 & $\mathrm{~N}$ & $\mathrm{Y}$ & $\mathrm{Y}$ \\
\hline & & & & & & & & & & (Fatigue) & (M \\
\hline
\end{tabular}




\begin{tabular}{|c|c|c|c|c|c|c|c|c|c|c|c|}
\hline 11 & $\mathrm{~F}$ & 29 & S & $130 / 87$ & 89 & 152 & 86 & 37.2 & $\begin{array}{l}\text { Y } \\
\text { (Asthma - mild, } \\
\text { controlled with } \\
\text { Ventolin) }\end{array}$ & $\mathrm{N}$ & $\begin{array}{l}Y \\
(H \epsilon\end{array}$ \\
\hline 12 & M & 43 & $\mathrm{Md}$ & $130 / 70$ & 80 & 173 & 81.5 & 27.4 & P (Allergies) & $\begin{array}{l}\text { P } \\
\text { (Weakness) }\end{array}$ & NP \\
\hline 13 & $M$ & 28 & $\mathrm{R}$ & $160 / 80$ & 40 & 192 & 93 & 25.2 & NP & (Heartburn) & NP \\
\hline 14 & M & 29 & Md & $122 / 81$ & 60 & 182 & 80 & 24.2 & $\begin{array}{l}\mathrm{P} \text { (History of } \\
\text { Jaundice and } \\
\text { inflammatory } \\
\text { condition) }\end{array}$ & $\begin{array}{l}P \text { (weight- } \\
\text { gain) }\end{array}$ & NP \\
\hline 15 & M & 52 & $\mathrm{Md}$ & $132 / 82$ & 68 & 184 & 102 & 30.1 & $\begin{array}{l}\mathrm{P} \text { (Allergies and } \\
\text { history of } \\
\text { musculoskeletal } \\
\text { condition) }\end{array}$ & NP & NP \\
\hline 16 & M & 44 & Md & $120 / 80$ & 72 & 188 & 90 & 25.5 & & & \\
\hline 17 & M & 23 & $\mathrm{R}$ & & $\begin{array}{l}\text { Y (history of } \\
\text { asthma and } \\
\text { musculoskeletal } \\
\text { condition) }\end{array}$ & $\begin{array}{l}\mathrm{P} \text { (Weight } \\
\text { loss, } \\
\text { fatigue } \\
\text { and } \\
\text { weakness) }\end{array}$ & $\begin{array}{l}\text { P } \\
\text { (numbness, } \\
\text { memory } \\
\text { loss and } \\
\text { headaches) }\end{array}$ & $\begin{array}{l}\text { No saliva } \\
\text { collected } \\
\text { at } \\
\text { Timepoint } \\
1\end{array}$ & & & \\
\hline 18 & M & 22 & $\mathrm{R}$ & $119 / 70$ & 64 & 180 & 100 & 30.9 & NP & $\begin{array}{l}\mathrm{P} \text { (weight } \\
\text { gain) }\end{array}$ & NP \\
\hline
\end{tabular}

Married $=$ Md, Relationship $=\mathrm{R}$, Single $=\mathrm{S}$, Divorced $=\mathrm{D}$

$\mathrm{Y}=$ Present, $\mathrm{N}=$ Not indicated

Table 3 - Challenges encountered by participants

\begin{tabular}{|llll|}
\hline $\begin{array}{l}\text { Category/ } \\
\text { item }\end{array}$ & Description & Rationale for design & Challenge encountered \\
\hline $\begin{array}{l}\text { Time of } \\
\text { the } \\
\text { therapy } \\
\text { session }\end{array}$ & $8-10$ am & $\begin{array}{l}\text { Participants were required to attend each consecutive } \\
\text { session at 8-9:30 am to achieve consistency and } \\
\text { reliability with the selected salivary biomarkers, as } \\
\text { these biomarkers show diurnal variation. }\end{array}$ & $\begin{array}{l}\text { Early morning appointments on two consecutive sessions } \\
\text { proved difficult for participants with early morning weekday } \\
\text { commitments. }\end{array}$ \\
\hline $\begin{array}{l}\text { Therapy } \\
\text { sessions }\end{array}$ & $\begin{array}{l}\text { Approximately } \\
\text { one hour, on two } \\
\text { consecutive days } \\
\text { (Monday and } \\
\text { Tuesday) }\end{array}$ & $\begin{array}{l}\text { As the intervention only ran for two days, having the } \\
\text { two sessions 24 hours apart lasting approximately } 1 \\
\text { hour was chosen for consistency. }\end{array}$ & $\begin{array}{l}\text { Arranging/organising two consecutive days were difficult for } \\
\text { some participants in light of their daily life commitments, i.e. } \\
\text { work. Furthermore, peak hour traffic and the clinic's location } \\
\text { off a busy main road added to difficulties. }\end{array}$ \\
\hline
\end{tabular}

Table 4 - Challenges encountered by the Osteopath researchers 


\begin{tabular}{|c|c|c|c|}
\hline $\begin{array}{l}\text { Category/ } \\
\text { item }\end{array}$ & Description & Reason/explanation & Challenge encountered \\
\hline $\begin{array}{l}\text { Recruitment } \\
\text { process }\end{array}$ & $\begin{array}{l}\text { Errors made } \\
\text { with initial } \\
\text { PSS-10 } \\
\text { scores for } \\
\text { three } \\
\text { participants } \\
(\# 1, \# 2 \text { and } \\
\# 4)\end{array}$ & $\begin{array}{l}\text { Osteopath researcher made an error in scoring, which led } \\
\text { to the inclusion of three participants who scored }<14 \text {. }\end{array}$ & $\begin{array}{l}\text { Data analysis and interpretation. } \\
\text { Three participants (\#1, \#2, \#4) were included in the study } \\
\text { although scored }<14 \text { PSS-10 due to nature and aim of the } \\
\text { study. This icl } \\
\text { - }\end{array}$ \\
\hline $\begin{array}{l}\text { Therapy } \\
\text { sessions }\end{array}$ & $\begin{array}{l}\text { Feasibility } \\
\text { regarding the } \\
\text { timing of the } \\
\text { sessions and } \\
\text { consecutive } \\
\text { days. }\end{array}$ & $\begin{array}{l}\text { Timing - 8-10 am decided to avoid diurnal variation } \\
\text { observed in the selected biomarkers. } \\
\text { Two consecutive days of the week: } \\
\text { The study was run as part of the student osteopaths' } \\
\text { research for their Masters, alongside completing clinical } \\
\text { hours, which is a compulsory component for their studies. }\end{array}$ & $\begin{array}{l}\text { The two available dates were mid-working week, which } \\
\text { became difficult for the student researchers to liaise with } \\
\text { their participants. In addition, due to the selection of the } \\
\text { times (8-10 am) the participants encountered challenges } \\
\text { with arriving on-time (between } 5-20 \text { minutes late). }\end{array}$ \\
\hline $\begin{array}{l}\text { Collection } \\
\text { of saliva } \\
\text { samples }\end{array}$ & $\begin{array}{l}\text { Difficulty } \\
\text { collecting } \\
\text { saliva from } \\
\text { participant, } \\
\# 5 \text { \#2, \#4, } \\
\# 10 \text { and \#12. }\end{array}$ & $\begin{array}{l}\text { It took nine minutes to collect and fill two cryotubes with } \\
\text { saliva for participant } \# 5 \text { at TP1. It took between } 5-7 \\
\text { minutes to collect saliva from participants } \# 4 \text { and } \# 10 \text { at } \\
\text { TP1. Participant \#4 had a coffee at } 6: 30 \text { am that morning. } \\
\text { Participant } \# 12 \text { could not produce any saliva. }\end{array}$ & $\begin{array}{l}\text { Participants who could not produce saliva as easily } \\
\text { appeared more stressed and agitated. Not producing } \\
\text { enough saliva may have caused participants to feel tense, } \\
\text { further increasing stress levels. }\end{array}$ \\
\hline
\end{tabular}

Timepoint $=\mathrm{TP}$

\section{Figures}

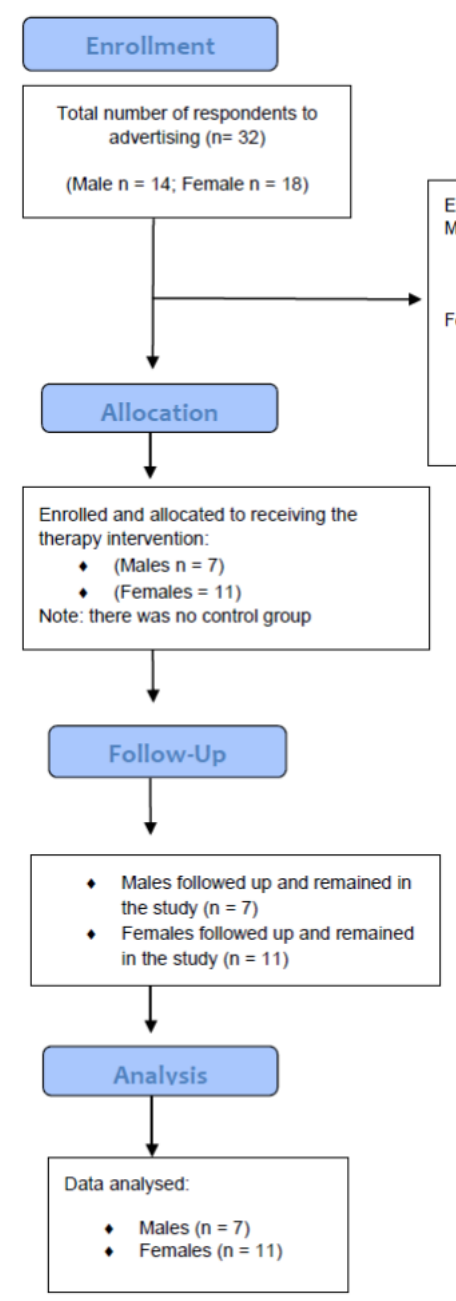

Figure 1.

Page 5/9 
Figure 1

Consort flow diagram showing participant flow at each stage of the trial (enrolment through analysis).

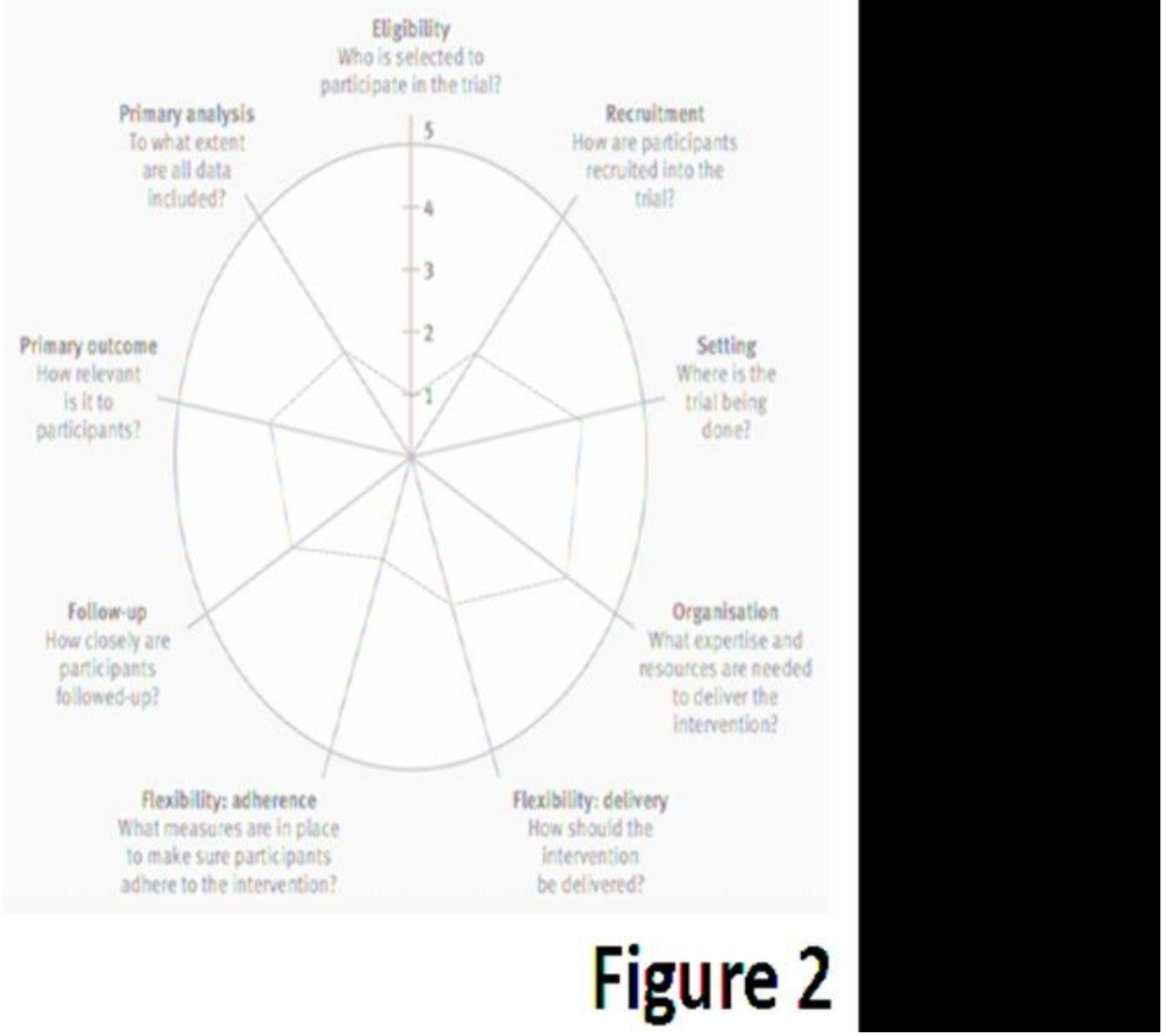

Figure 2

PRECIS-2 Wheel score for the study. 'Setting and organization' rated as rather pragmatic, 'eligibility' rated as very exploratory; 'recruitment', 'primary analysis' and 'flexibility: adherence' read as rather exploratory; 'flexibility: delivery', 'follow-up' and 'primary outcome' rated as mixed pragmatic and exploratory. Total score was 26 


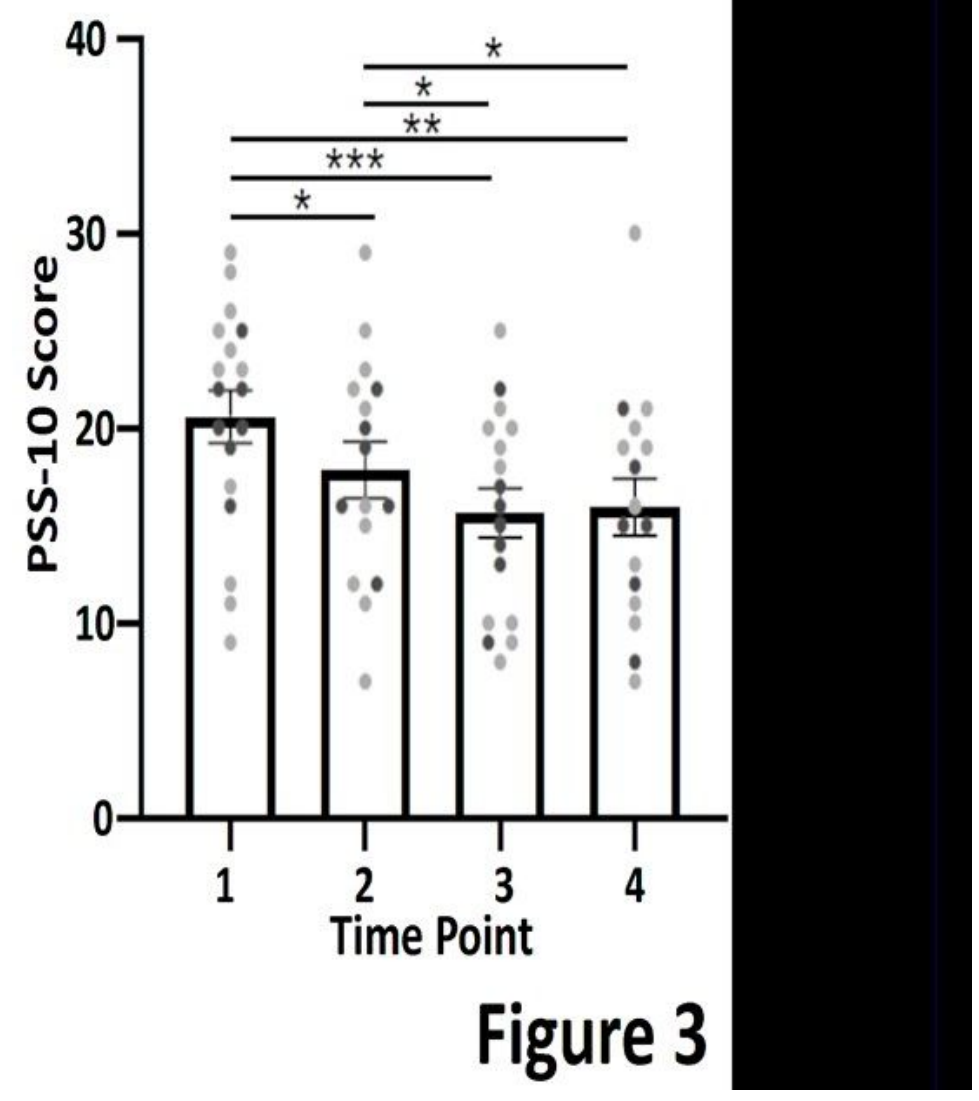

Figure 3

Bar graph showing the mean PSS-10 scores from all 18 participants at time point 1, 2, 3 and 4 . Error bars are SEM. * represents adjusted $p$ value $<0.05$, ** represents adjust $p$ value $0.01, * \star \star *$ represents adjusted $p$ value $<0.001$. Pink data values denote female participants and blue data values denote males. 
A.

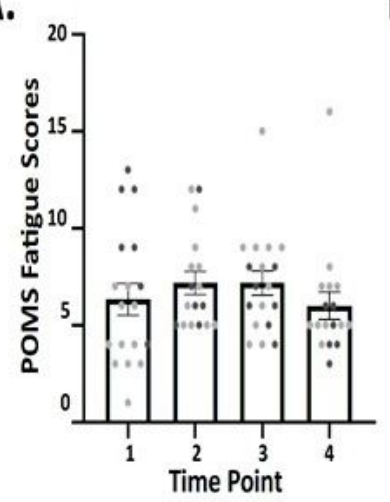

C.

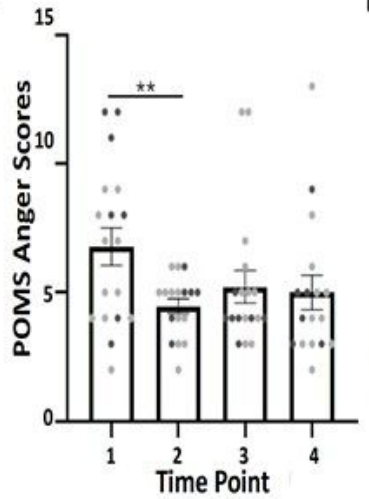

B.

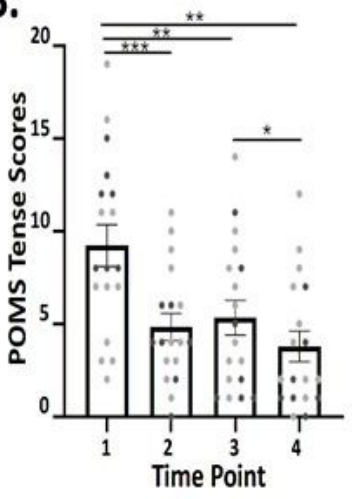

D.

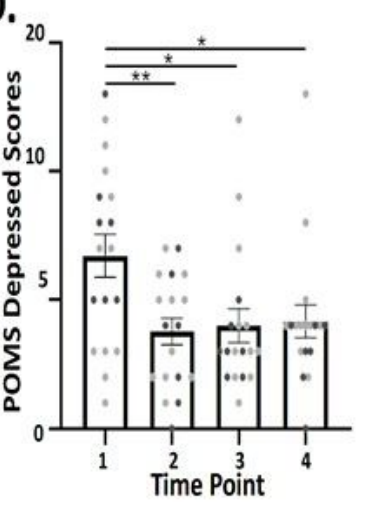

Figure 4

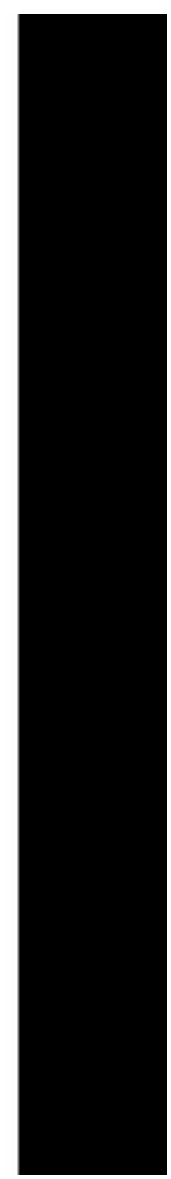

Figure 4

Bar graphs showing the mean POMS scores from all 18 participants for fatigue, tense, anger and depressed subscales. POMS fatigue scores (A), POMS tense scores (B), POMS anger scores (C), and POMS depressed (D) scores at time point 1, 2, 3 and 4. Error bars are SEM. * represents adjusted $p$ value $<0.05$, ** represents adjust $p$ value $0.01, * * *$ represents adjusted $p$ value $<0.001$. Pink data values denote female participants and blue data values denote males.
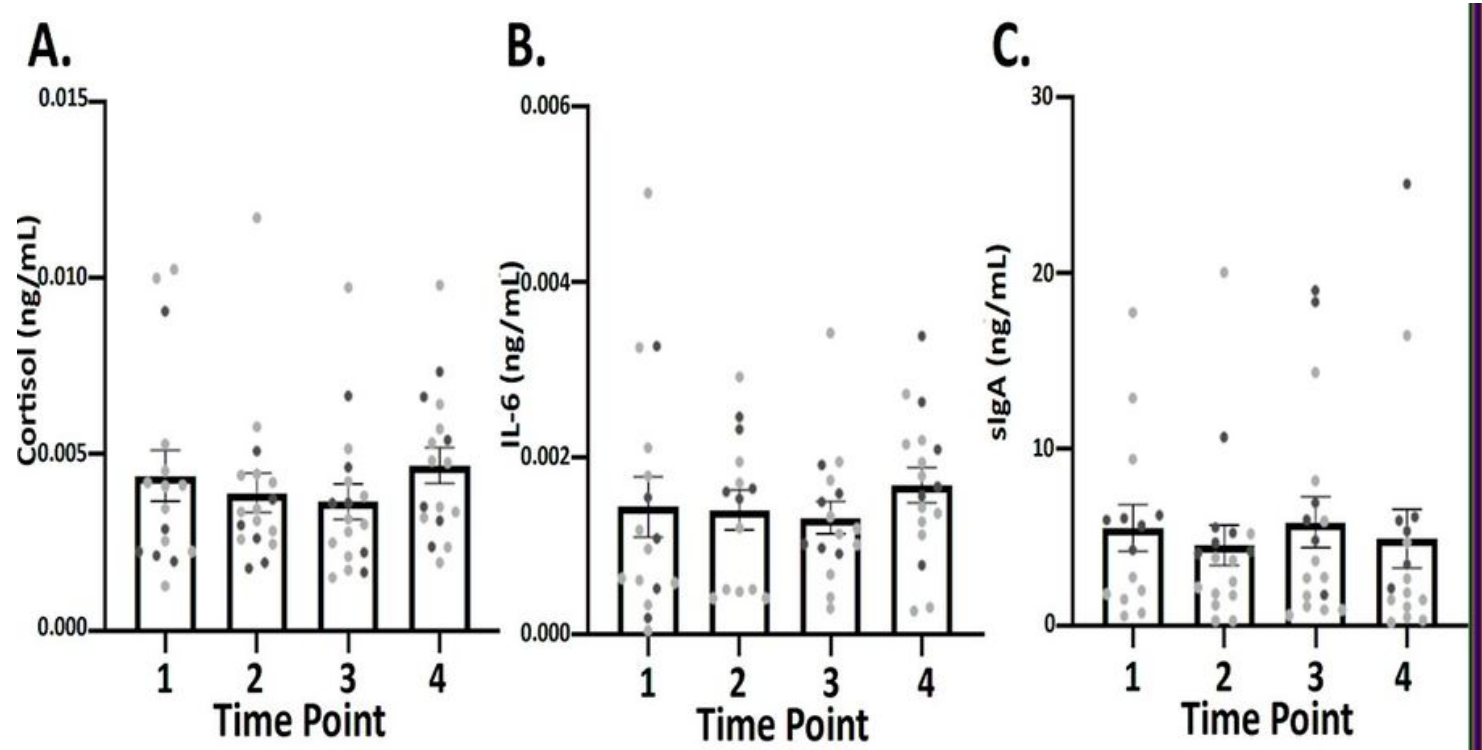

Figure 5

Figure 5

Bar graphs showing the mean concentration of stress related saliva biomarkers from all 18 participants. The concentration of cortisol (A), IL-6 (B) and slgA (C) at time point 1, 2, 3 and 4 . Pink data values denote female participants and blue data values denote males.

\section{Supplementary Files}


This is a list of supplementary files associated with this preprint. Click to download.

- Rahieretal.2020CONSORT2010reportingchecklist.pdf

- SupplementaryFigure1.tif

- SupplementaryFigure2.tiff

- SupplementaryFigure3.tif

- SupplementaryFile10SupplG0Tinfo.docx

- SupplementaryFile11scriptedPE.docx

- SupplementaryFile12PEpamphlet.docx

- SupplementaryFile1CONSORT2010checklist.docx

- SupplementaryFile2Advert.docx

- SupplementaryFile3MedHistory.docx

- SupplementaryFile4Protocolsteps.docx

- SupplementaryFile5PIS.docx

- SupplementaryFile6Consentform.docx

- SupplementaryFile7PSS10.docx

- SupplementaryFile8POMS.docx

- SupplementaryFile9GOTs.docx 